\title{
Ligand coupling reactions of hypervalent species
}

\author{
Shigeru Oae \\ Institute of Heteroatom Chemistry, 2509 Hirao Mihara-cho \\ Minamikawachi-gun 587 Japan.
}

\begin{abstract}
The concept of ligand coupling is explained and the actual examples of many important reactions in which not only sulfur and phosporus centered hypervalent species, but iodine, silicon and copper centered hypervalent ones are presented. It was also mentioned that many other reactions in which the central metal atoms in the nickel triad elements are considered to behave as the catalytic site for ligand coupling reaction, such as the Wäker process and the Heck reaction.
\end{abstract}

Ever since we found the following reaction,(1) we have extended our concept of ligand coupling(2) and have so many examples of these or similar reactions. However, there were prior many reactions to enable us to postulate the concept.

A typical example of a three-centered, four electron bond, called a hypervalent bond by Musher,(3) can be found in the substance in the structure of quternary florinated sulfur as demonstrated by X-ray analysis by Rundle et al.(4) and Pimentel, (5) in the following figure. (Fig. 1)
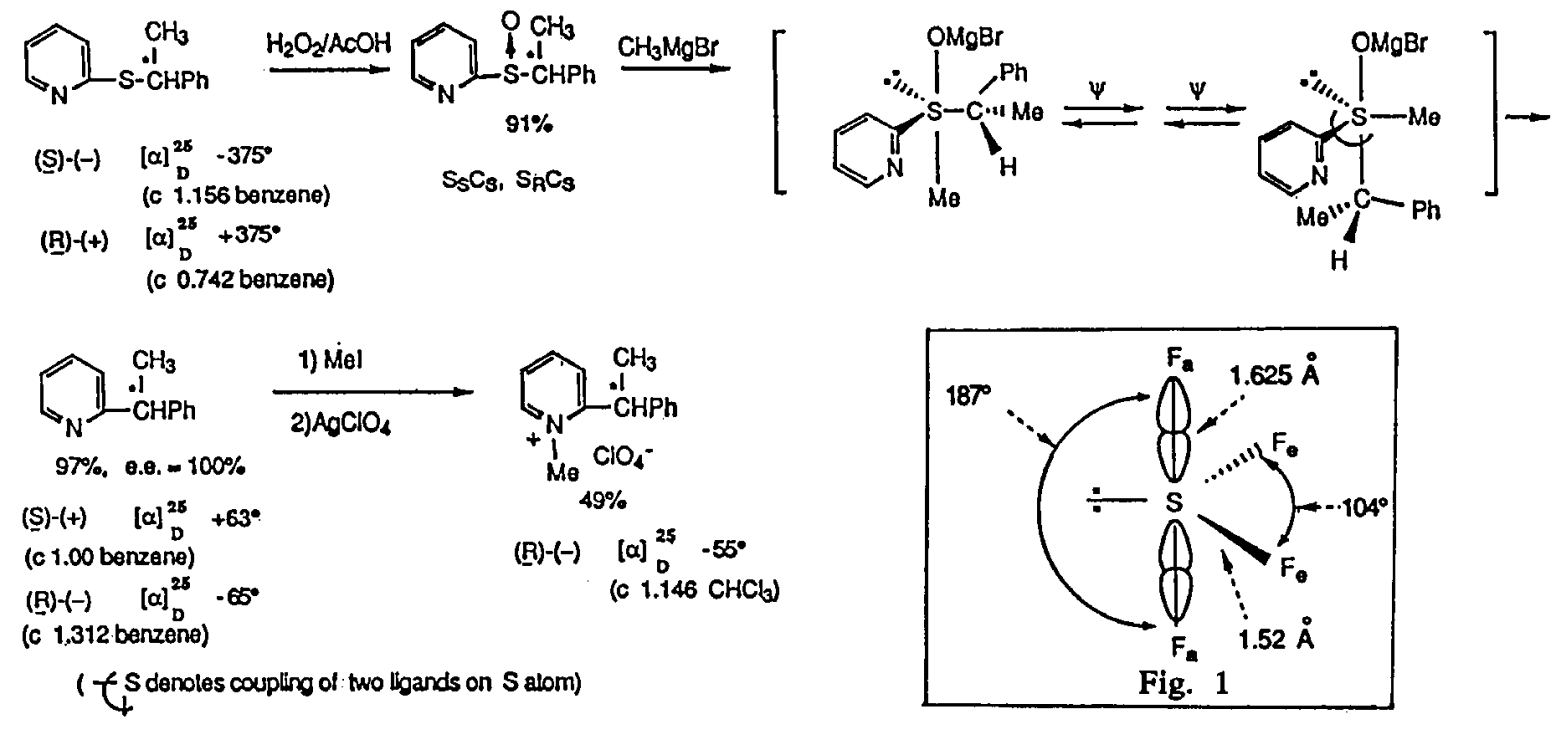

The central atom in a hypervalent species is valence-shell-expanded and tends to to extrude one pair of electrons to the normal valency of an octet. This is the driving force of the reaction and there are three conceivable ways for hypervalent species to collapse to form stable octet species; self-decomposition as exemplified by the wellstudied Wittig reaction,( 6)ligand exchange, $(7,8)$ and ligand coupling.(9-13) It is well known that the equatorial ligands form $\mathrm{sp}^{2}$ and the axial linkages assume 
p-orbitals. The axitial bonds are longer and hold the electronegative substituents, as halogen while the equatorial ones are held with aromatic, olefinic and the negative groups, such as sulfoxide S-O bond In dealing with the hypervalent species, one has to consider another physicochemical phenomenon, such as the tropological transformation, as BPR or TR. The following is the results of stereochemistry, when these hypervalent species are handled.

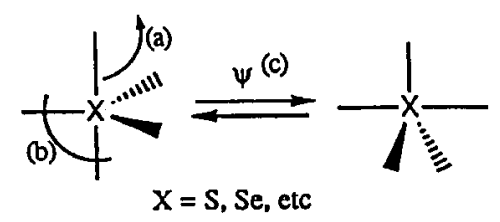

\begin{tabular}{ccc}
\hline & Chemical Phenomenon & Stereochemistry \\
\hline (a) Ligand Exchange & inversion \\
+ (c) & retention \\
(b) Ligand Coupling & retention \\
(c) Topological Transformation & retention or \\
(BPR or TR) & racemization \\
\hline
\end{tabular}

One of the driving force of ligand coupling is the following effective overlapping, shown by the sulfur-centered reaction, as illustrated below.

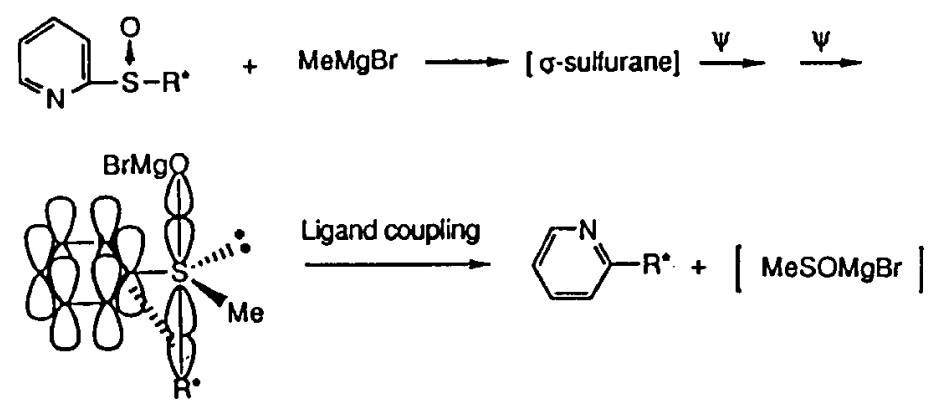

Meanwhile, the expression "reductive elimination" and "oxidative addition" are frequently used in organometallic chemistry. This term is also a mere description of the phenomenon and lacks mechanistic significane. The only two examples are show below.(14-15)

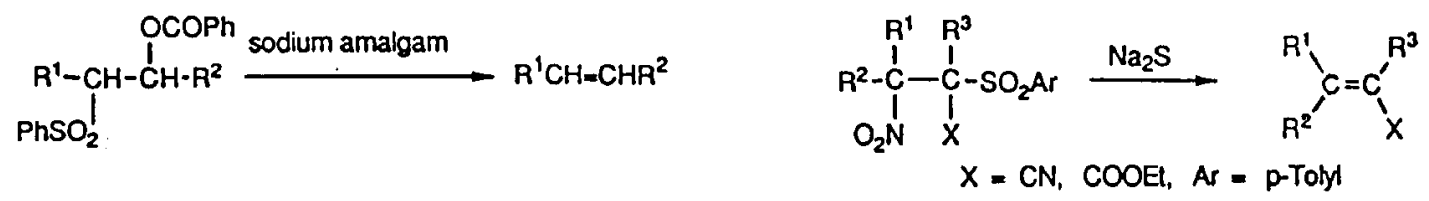

Of cause, prior to this assumption, there are many, many experiments and ideas.(1639)

Another example of stereochemistry is shown below.(40) There is another example

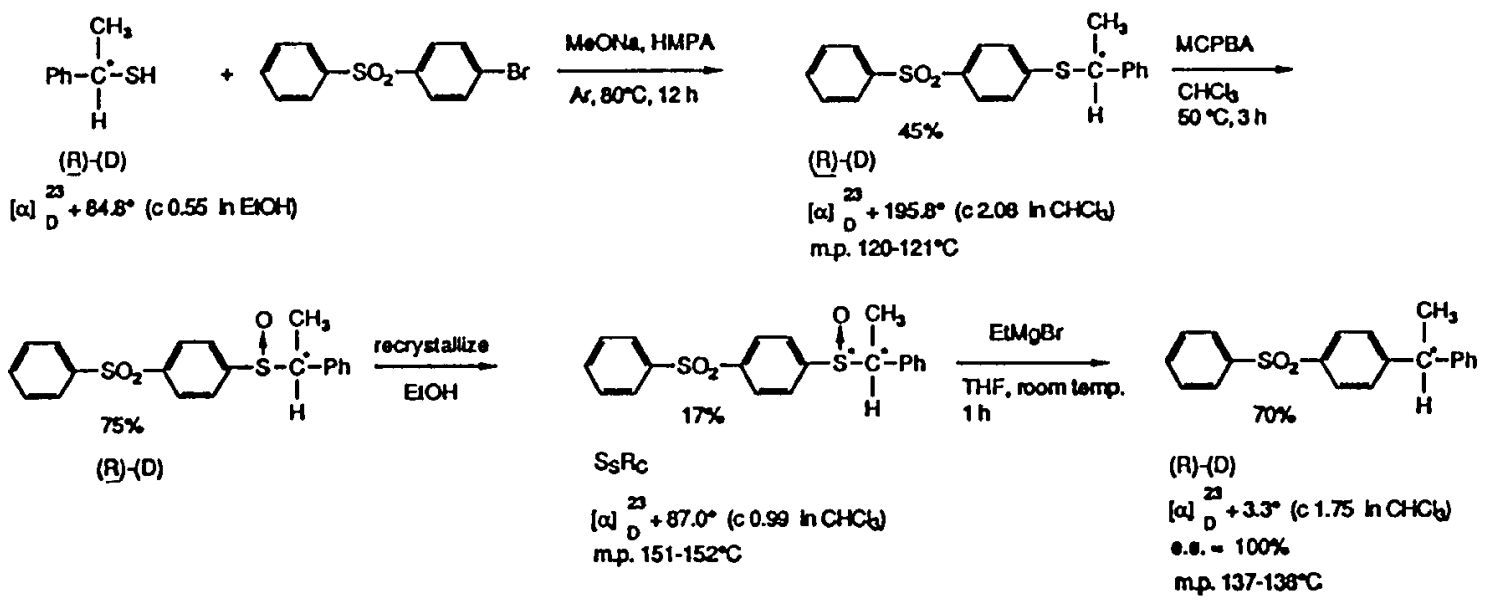


is between optically active1-phenylethyl 2-quinolyl sulfoxide and methylmagnesium bromide to afford completely retained1-phenylethyl-2-quinoline in a high yield.(41) In addition, there are a number of stereochemistry of geometetrical isomers in the following ligand coupling reactions.(42)
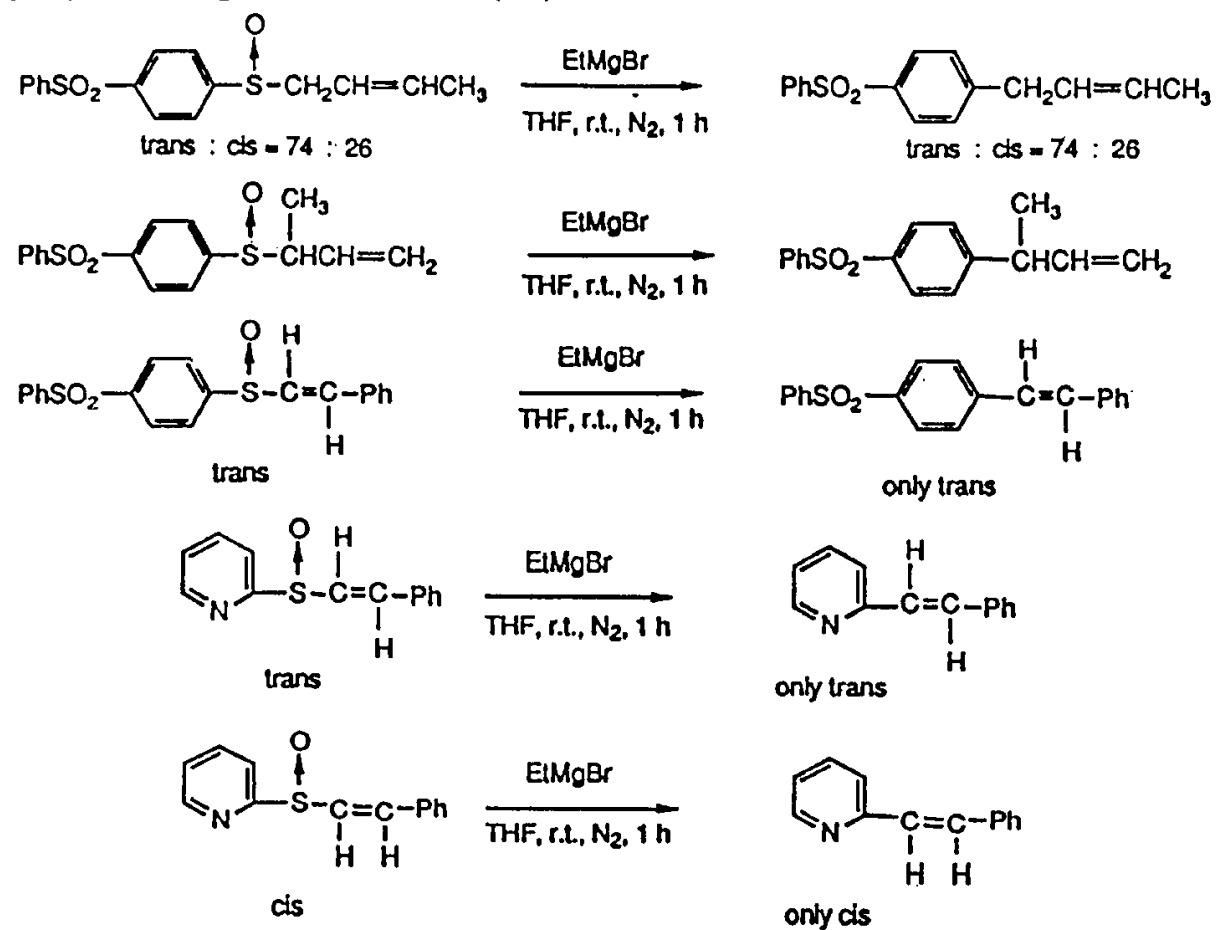

only trans

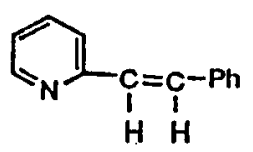

only dis

In all the journals, we have found good many examples of ligand coupling reaction, but only a few which we or our friends carried out, are presented.(43-50)

We turn next to the ligand coupling on phosphorus atom. The sterochemistry of the ligand coupling on hypervalent phosphorus atom was carried out by Seyferth et al. accidentally in the following reactions.(51) The yields are good while the stereochemistry in both cases are nearly perfect.

$\mathrm{Pr}_{4} \mathrm{P}^{+} \mathrm{Br}^{-}+\mathrm{CH}_{3}{ }^{-} \mathrm{Ll} \longrightarrow \mathrm{CH}_{3}{ }_{\mathrm{Ph}} \mathrm{PH}_{4} \mathrm{P}^{+} \mathrm{Br}^{-}+\mathrm{CH}_{3}-\mathrm{Hl}^{-} \longrightarrow \mathrm{CH}_{3} \longrightarrow \mathrm{Ph}$

Many examples of the ligand coupling involving 2-pyridyl groups are summarized in the chart, as shown below.(52-55) All these coupling reactions do not require

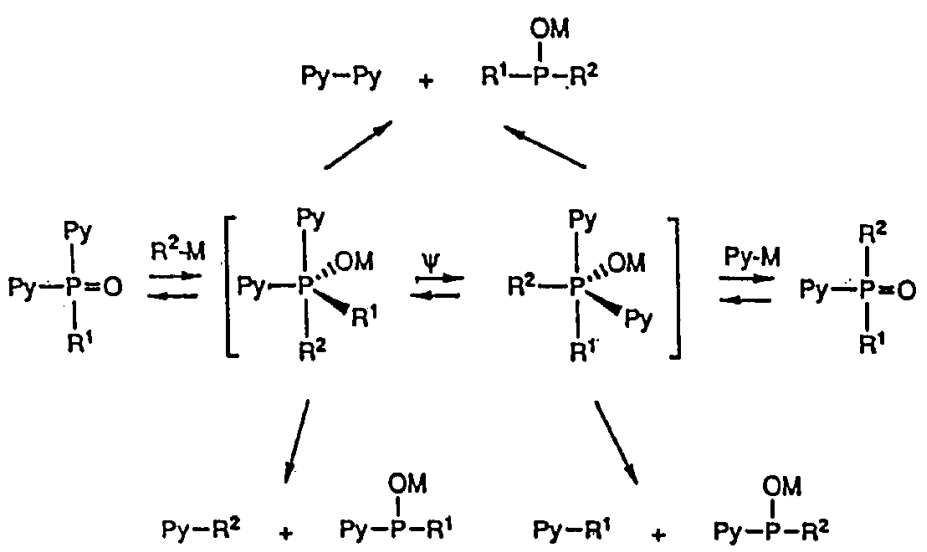

- $\mathrm{Py}=2$-pyridyl; $\mathrm{R}^{1}=2$-pyridyl, $\mathrm{Ph} ; \mathrm{R}^{2}=\mathrm{Me}, \mathrm{Ph}, \mathrm{PhCH}_{2}, 4-\mathrm{M}_{\theta} \mathrm{C}_{6} \mathrm{H}_{4} \mathrm{CH}_{2} ; \mathrm{M}=\mathrm{Li}, \mathrm{MgX}$. 
any alkaline condition(56) nor quaternary phosphorus compounds, as shown in the following reaction.

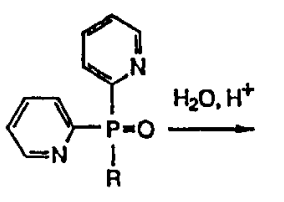<smiles>[2H]P1(O)(c2ccccn2)C2CCC(C2)CC1(O)PC</smiles>

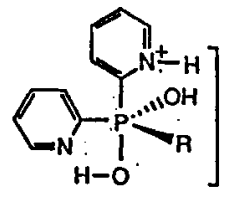

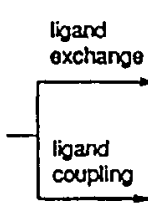<smiles>[2H]P(=O)(O)c1ccccn1</smiles>

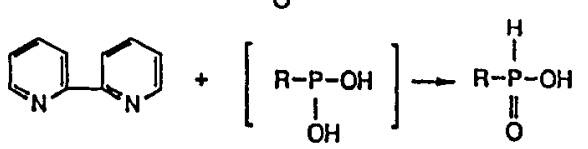

The uses of phosphrus trichloride, phosphorus oxychloride and thionyl chloride were found to undergo coupling reaction with many heterocycles.(57)(Table. I) The following examples are also interesting to note.
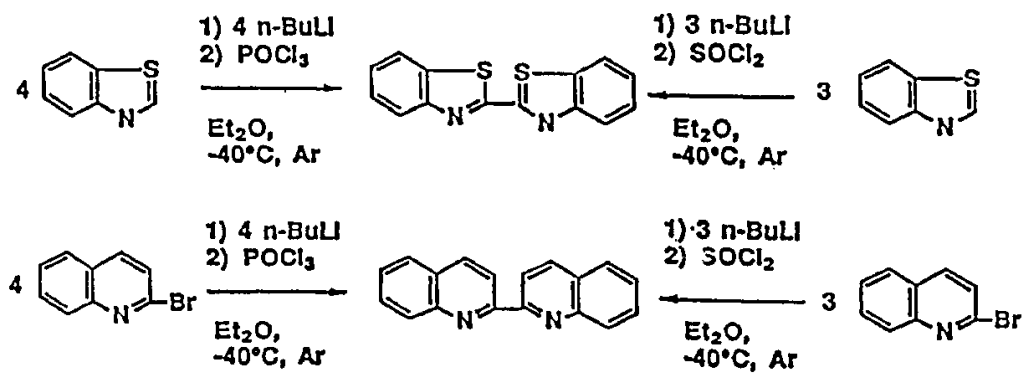

The involvement of 2-pyridyllithium in the ligand coupling is known in the following example.(58)

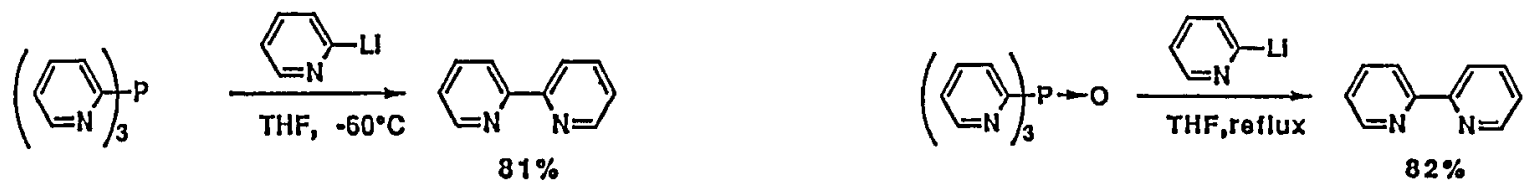

Meanwhile, the following electrophilic substutution by chlorine was found to occur.(59)

$$
\left.\left({ }_{-N}\right)_{3} \frac{\mathrm{Cl}_{2}}{\mathrm{MeCN}}\left({ }_{-N}\right)_{3}\right)_{\text {reflux }} \frac{\mathrm{MeOH}}{\text { refle }}
$$

The use of the protic solvent is preferable. Chlorination, bromination, deuteration and diazocoupling are achieved,(60) as shown below. We believe that these are typical electrophilic substitutions, since the phosphrus atom in such a pentacoordinate species, especially 3-position of the axial pyridine ligand, is susceptical to the electrophilic substitution.
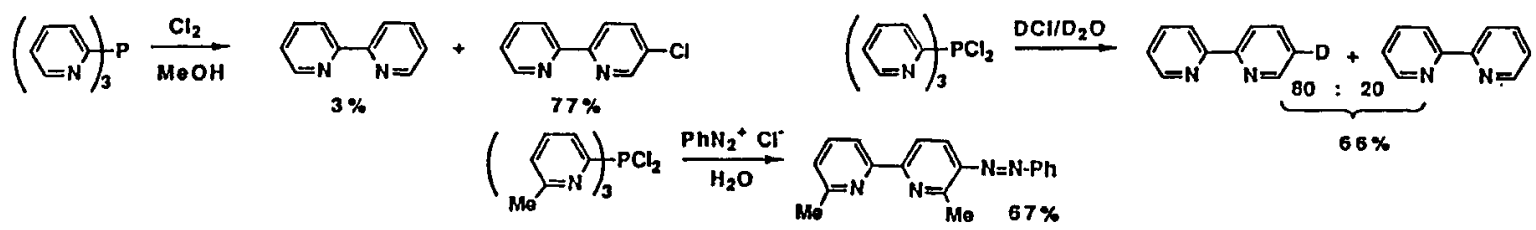
We then turn to the hypervalent silicon species and only one example is sited below. $(61,62)$
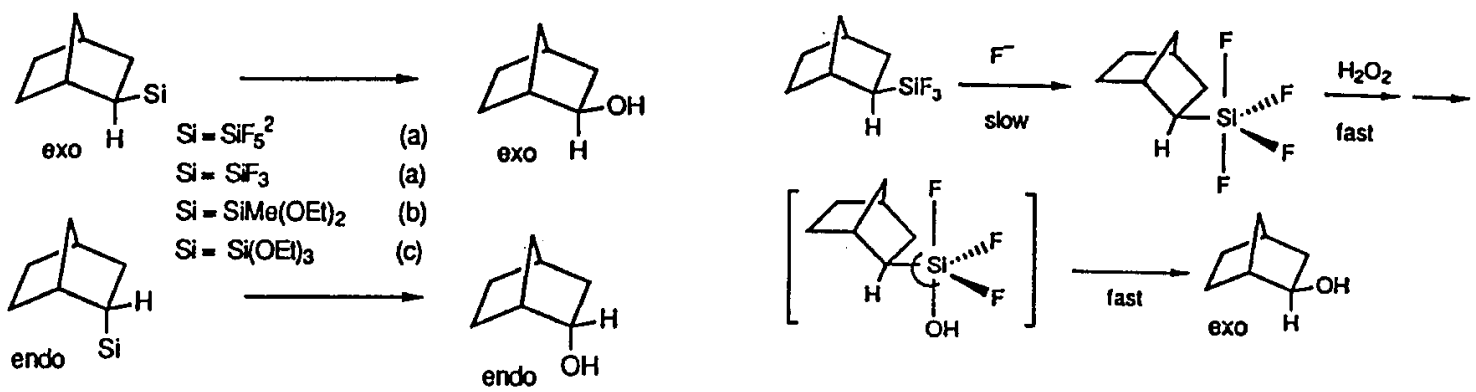

- Reaction conditions: (a) MCPBA/DMF/room temperature; (b) $30 \% \quad \mathrm{H}_{2} \mathrm{O}_{2} / \mathrm{KHF}_{2} / \mathrm{DMF} / 60{ }^{\circ} \mathrm{C}$; (c) $30 \% \quad \mathrm{H}_{2} \mathrm{O}_{2} / \mathrm{NaHCO}_{3}$ / $\mathrm{MeOH} / \mathrm{THF} / 60^{\circ} \mathrm{C}$.

What about the central iodine atom? There are so many and I do not want to go in detailed discussions but cite only a few examples. $(63,64)$

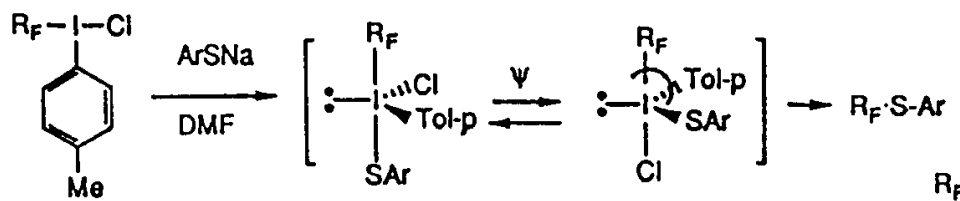

$R_{F}:$ Perfluoroalkyl group $\mathrm{R}_{\mathrm{F}}-\mathrm{Ph}$

$\mathrm{R}_{\mathrm{F}}-$ I $_{\mathrm{Ph}}$<smiles>Oc1ccc(F)cc1</smiles>

As we said earlier(2,30), many reactions involving organic copper reagents, scuh as the Ullmann type reaction shown below,(66) and the following one is a few typical

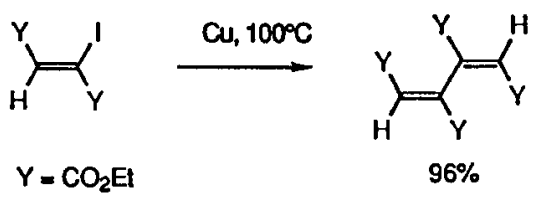

example of the fast ligand coupling within the hypervalent copper(III) intermedate after a typital $\mathrm{S}_{\mathrm{N}} 2$ process, as shown below.(67)

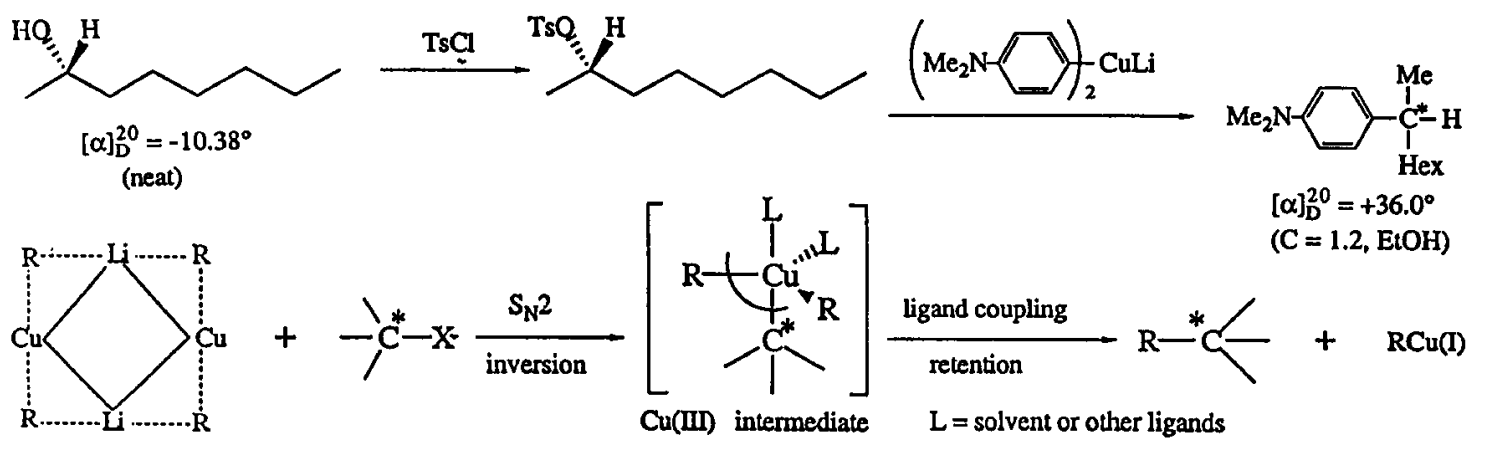

There are a number of examples of ligand coupling within hypervalent species of the nickel triad.(68) We are going to present only one stereospecific example to form the trans-acetoxoxypalladium, eventually.giving the final trans product.(69)

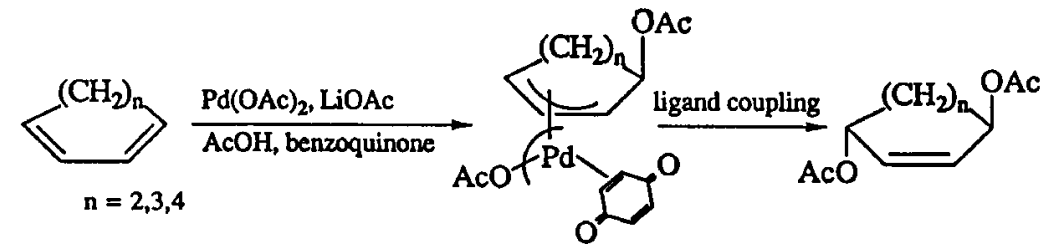


Another example is a typical Ullmann cross-coupling reaction shown below.(70)<smiles>O=[N+]([O-])c1ccccc1-c1ccc(-c2ccccc2)cc1</smiles>

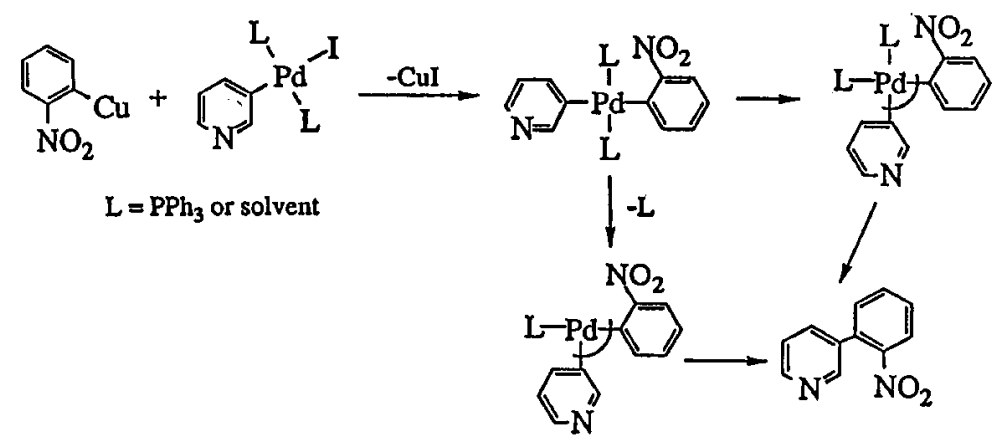

Acknowledgement. Many colleagues have collaborated in pursuing this work. Professors N. Furukawa, Y. H. Kim who is the chief organizer of this ICHAC-4 T. Aida, T. Yoshimura when I was at Osaka City University. In the University of Tsukuba, Professor N. Furukawa, Dr. T. Kawai and when I was in Okayama University of cience Professors S. Wakabayashi and J. Uenishi and Dr.T.Takeda. After that, Professors Y. Uchida, K. Negoro and M. Yoshihara have done many important works; especially Dr. Y. Inubushi and Mr. H. Ishihara have done so much contributions in this Institute. I can by no means forget many useful comments and opinions of my friends who have kindly suggested in pursuing the interesting works.

\section{REFERENCES}

1. S. Oae, T, Kawai, N. Furukawa, Teterahedron Lett., 25, 65 (1984); S. Oae, T. Kawai, N. Furukawa, F. Iwasaki, J. Chem. Soc.,Perkin Trans., $\underline{3}$ (1987) 405.

2. S. Oae, Croat. Chem. Acta, 59, 129 (1986); S. Oae, Phosphorus and Sulfur, 27, 13 (1986).

3. J. I. Musher, Angew. Chem. Int. Ed. Engl., 8, 54 (1969).

4. R. J. Hoh, R. E. Rundle, J. Am. Chem. Soc., 71, 48 (1951).

5. G. C. Pimentel, J, Chem. Phys., 19, 446 (1951).

6. G. Wittig, Pure Appl. Chem., 9, 245 (1964); G. Wittig, H, Fritz, Liebig Chem., 577, 39 (1952).

7. R. R. Holmes, "Pentacoordinated Phosphorus", ACS Monograpfs 175 and 176 Am. Chem. Soc.. Washington, D.C., Vols.,I and III (1977).

8. S. Oae, "Organic Sulfur Chemistry-Structure and Mechanism" CRC Press Florida (1991), Chapeter 5; T. Kawai, N. Furukawa, S. Oae, Tetrahedron Lett. 25, 2549 (1984).

9. Y. H. Khim, S. Oae, Bull. Chem. Soc. Jpn., 42, 1968 (1969).

10. B.M. Trost, R. La Rochelle, R. C. Atkins, J. Am. Chem. Soc., 91, 2175 (1969); 93, 6077 (1972).

11. M. Hori, T. Kataoka, H. Shimizu, Chem. Pharm Bull., 22, 1711, 2004, 2014, 2020, 2030 (1974).

12. W. A. Sheppard, J. Am. Chem. Soc., 93,5597 (1973) 
13. T. Aida, N, Furukawa, S. Oae, Chem. Lett. 805 (1973), 29 (1975); J. Chem. Soc., Perkin Trans. II 1438 (1976).

14. P. J. Kocienski, B. Lythgoe, I. Waterhuse, J. Chem. Soc., Perkin Trans. 1, 1045 (1980).

15. N. Ono, R. Tamura, J. Hayami, A. Kaji, Tetrahedron Lett., 763 (1978).

16. S. Oae, T. Aida, N. Furukawa, Chem. Pharm. Bull.,3011 (1975); Int'l. J. Sulfur Chem., 401 (1973).

17. S. Oae, T. Yoshimura, N. Furukawa, Bull. Chem. Soc. Jpn., 45, 2019 (1972).

18. T. Takeda, Ph.D. Thesis, Okayama University of Science (1989).

19. S. Oae, T. Takeda, T. Kawai, N. Furukawa, Phosphorus and Sulfur, 34, 133;

T. Kawai, Y. Kodera, N. Furukawa, S. Oae, M. Ishida, T. Takeda, S. Wakabayashi, ibid., 34, 139 (1987).

20. S. Oae, "Yukiokagaku / Organic Sulfur Chemistry", Kagaku Dojin, Kyoto, Chapter 3, 4 (1982).

21. S. Oae, N. Furukawa, "Sulfilimenes and Related Derivatives" ACS Monogrph 179, Am. Chem. Soc. (1983).

22. S. Oae, "Historical Development of Sulfur Bonding" in "Organic Sulfur Chemistry", edited by F. Bernardi, I. G. Csizmadia, A. Mangini, Elesevier, Amsterdam (1985).

23. S. Oae, T. Kawai, N. Furukawa, Phosphorus and Sulfur, 34,123 (1987).

24. S. Wakabayashi, M. Ishida, T. Takeda, S. Oae, Tetrahedron Lett. 29, 4441 (1988).

25. S. Oae, T. Takeda, S. Wakabayashi, Heterocycles, 28, 99 (1989).

26. S. Wakabayashi, T. Takeda, Y. Kubo, J. Uenishi, S. Oae, Bull. Chem. Soc. Jpn., 62, 3848 (1989).

27. S. Wakabayashi, Y. Kubo, T. Takeda, J. Uenishi, S. Oae, Bull. Chem. Soc. Jpn., 62, 2338 (1989).

28. J. Uenishi, T. Tanaka, S. Wakabayashi, S. Oae, H. Tsukube, Tetrahedron Lett., 31,4625 (1990).

29. S. Oae, N. Furukawa, "Heteroaromatic Sulfoxides and Sulfones: Ligand Exchange and Coupling in Sulfuranes and Ipso-Substitutions",in Advances in Heteroacyclic Chem. (Ed.) by A. R. Katrizky (Academic Press), 48, 1-63 (1990).

30. S. Oae, Reviews on Heteroatom Chem., 1 , 304, (1988).

31. S. Oae, Y. Uchida, Reviews on Heteroatom Chem., 2 , 76 (1989).

32. S. Oae, Reviews on Heteroatom Chem., 4, (1991).

33. J. Uenishi, T. Tanaka, K. Nishiwaki, S. Wakabayashi, S. Oae, H. Tsukube, J. Org. Chem., 58, 4382 (1993).

34. H. Tsukube, J. Uenishi, H. Higaki, K. Kikukawa, T. Tanaka, S. Wakabayashi, S. Oae, J. Org. Chem., 58, 4389 (1993).

35. S. Wakabayashi, Y. Kiyohara, S. Kameda, J. Uenishi, S. Oae, Heteroatom Chem., 1, 225 (1990).

36. K. S. Kim, I. B. Jung, Y. H. Kim, S. Oae, Tetrahedron Lett., 30, 1087 (1989).

37. S. Oae, Y. Inubushi, M. Yoshihara, Heteroatom Chem. 4, 185 (1993); $\underline{5}$, 223 (1994); S.Oae, Y. Inubushi, M. Yoshihara, Phosphorus, Sulfur and Silicon, (in print).

38. S. Oae, H. Ishihara, M.Yoshihara, Zhur. Org. Khim.,(in print). 
39. Y. Inubushi, Ph. D. Thesis, Kinki University, (1995).

40. S. Oae, T. Takeda, S. Wakabayashi, F. Iwasaki, N. Yamazaki, Y. Katsube, J. Chem. Soc., Perkin Trans, 2, 273 (1990).

41. J. Uenishi, A. Yamamoto, T. Takeda, S. Wakabayashi, S. Oae, Heteroatom Chem., 3, 73 (1992).

42. S Oae, T. Takeda, S. Wakabayashi, Tetrahedron Lett.,29, 4445 (1988).

43. C. R. Johnson, C. W. Schroeck, J. R. Shauklin, J. Am. Chem. Soc. 95,7424 (1973).

44. J. Kiji, M. Iwamoto, Tetrahedron Lett., 2749 (1966).

45. S. Oae, Y. Tsuchida, K. Tsujihara, N. Furukawa, Bull. Chem. Soc. Jpn., 45 , 2856 (1972).

46. H. Yoshida, H. Teketani, T. Ogata, S. Inakawa, Bull. Chem. Soc. Jpn., 49 , 3124 (1976), 50, 3302 (1977).

47. T. Aida, Ph. D. Thesis at Osaka City University, (1974).

48. R. L. Smorada, W. E. Truce, J. Org. Chem., 44, 3444 (1979).

49. G. H. Wiegant, W. E. McEwen, J. Org. Chem., 33, 2971 (1966).

50. S. Ogawa, private communications.

51. D. Seyferth, J. Fogel, J. K. Heeren, J. Am. Chem. Soc., 88, 2207 (1966).

52. Y. Uchida, K. Onoue, K. Tada F. Nagano, S. Oae, Tetrahedron Lett., $\underline{30}$ 567 (1989).

53. Y. Uchida, H. Kozawa, S. Oae, Tetrahedron Lett., 30, 6365 (1989).

54. Y. Uchida, Y. Takaya, S. Oae, Heterocycles, 55, 347 (1990).

55. Y. Uchida, K. Onoue, N. Tada, F. Nagao, H. Kozawa, S. Oae, Heteroatom Chem., 1, 295 (1990); Y. Uchida, H. Kawai, H. Masauji, S. Oae, Heteroatom Chem., 4, 421 (1993).

56. G. R. Newkome, D. C. Hager, J. Am. Chem. Soc, 100, 5567 (1978).

57. Y. Uchida, N. Echikawa, S. Oae, Heteroatom Chem, 5, 409 (1994).

58. Y. Uchida, R. Kajita, Y, Kawasaki, S. Oae, Phosphorus, Sulfur and Silicon, 93(1-4) \& $\underline{94}(1-4) 403$ (1994).

59. Y. Uchida, R. Kajita, Y. Kawasaki, S. Oae, Tetrahedron Lett., 36, 4077 (1995).

60. Y. Uchida, S. Oac, XIII ICPC Abstract, Jerusalem, July (1995).

61. K. Tamao, T. Hayashi Y. Ito, Nippon Kagaku Kaishi, 509 (1990); Tetrahedron Lett., 31, 2925 (1990).

62. K. Tamao, T. Hayashi, Y. Ito, unpublished, cited in 61.

63. V. V. Lyalim, V. V. Orda, L. A. Alekseeva, L. M. Yagpolskii, Zhur. Org.

Khim., 7, 1473 (1971); Synthesis, 835 (1978).

64. T. Umemoto, Y. Kuriu, Tetrahedron Lett., 22, 5197 (1981); Bull. Chem. Soc. Jpn.,57, 3361 (1984).

65. T. Umemoto, Yuki Gosei Kagaku Kyokaishi, 41, 251 (1981).

66. T. Cohen, T. Poeth, J. Am. Chem. Soc., 94, 4363 (1972).

67. K. Negoro, S. Oae, Reviews on Heteroatom Chem., 9123 (1993).

68. K. Negoro, S. Oae, Reviews on Heteroatom Chem., 13, (in print).

69. J.-E. Backvall, Acc. Chem. Res., 16, 335 (1983).

70. N. Shimizu,T. Kitamura, K. Watanabe,T. Yamaguchi, H. Shigyo, T. Ohta, Tetrahedron Lett., 34, 3421 (1993). 